\title{
Review: oral melatonin reduces jet lag in air travellers
}

Herxheimer A, Petrie KJ.Melatonin for preventing and treating jet lag. Cochrane Database Syst Rev 2001;(1):CD001520 (latest version 22 Aug 2000).

\section{QUESTION: In people who travel by air across several time zones, can oral melatonin prevent or treat jet lag (daytime fatigue, sleep disturbances, loss of mental efficiency, weakness, and irritability)?}

\section{Data sources}

Studies were identified by searching the Cochrane Controlled Trials Register, Medline, EMBASE/Excerpta Medica, and PsycLIT with the terms melatonin, jet-lag, jet lag, aviation, air travel, and airtravel. Specific studies were identified in SciSearch, 2 journals (Aviation, Space and Environmental Medicine and Sleep from 1986 to 1999) were hand searched, bibliographies of relevant studies were scanned, and authors of studies were contacted to identify further studies. Data and case reports of adverse effects of melatonin were also sought.

\section{Study selection}

Randomised controlled trials were selected if airline passengers, airline staff, or military personnel were studied; oral melatonin was compared with placebo or other medication; drugs were taken before, during, or after travel or a combination of times related to travel; and outcome data were provided.

\section{Data extraction}

Data were extracted on study quality and participants, timing and dose of melatonin and other study medications, flight information, and outcomes of subjective ratings of jet lag and its components (fatigue, daytime tiredness, onset of sleep at destination, onset and quality of sleep, psychological functioning, duration of return to normal, and measures indicating the phase of circadian rhythms)

\section{Main results}

10 trials met the inclusion criteria; 1 was excluded because of design weaknesses. All trials compared melatonin with placebo. Melatonin was taken at the same clock time after arrival at the destination (close to bedtime at the destination). All trials evaluated treatment for $>2$ days; none examined shorter duration's of treatment. 9 of the trials individually showed a reduction in symptoms of jet lag in favour of melatonin. Meta-analysis showed that the weighted mean difference in the global jet lag rating scale (0 to 100) was $38(95 \%$ CI 35 to 40). 1 study showed that taking melatonin before and after travel was no better than taking it only after arrival. Another study showed that jet lag was worse after eastward flights than westward flights. Both passengers and airline staff showed improvement with melatonin. Daily doses between 0.5 and $5 \mathrm{mg}$ were similar in effectiveness, except that on the higher dose people fell asleep more quickly. Doses $>5 \mathrm{mg}$ were no more effective than doses $\leqslant 5 \mathrm{mg}$. Slow-release melatonin $(2 \mathrm{mg})$, was less effective than fast-release tablets. Adverse effects were few but were not systematically measured.

\section{Conclusion}

Oral melatonin reduces jet lag in air travellers.

\section{COMMENTARY}

Jet lag is a universal phenomenon and a cause of discomfort and distress for both vacationers and business travellers. 4 strategies have been used to address jet lag: sleep (timing and induction), light exposure carefully timed to help with the readjustment of the body clock, activity, and melatonin. The acronym SLAM has been applied to these strategies. ${ }^{1}$ The review by Herxheimer and Petrie considers the limited research on melatonin as an agent for preventing jet lag and cleverly evaluates the available studies from several perspectives, including the use of placebo in studies, dosage of melatonin, direction of flight, and duration of treatment. The authors document the limitations of the studies but distill clear results that can be used clinically.

The following findings were included: 1 in 2 people receiving melatonin was likely to benefit; high-dose melatonin prompted sleep induction and decreased fatigue the next day more than low-dose melatonin; cognitive tests done after waking by those receiving melatonin had half as many errors as those receiving placebo; and receiving melatonin before and after the flight was no better than receiving it only after the flight. Specific cautions pertaining to the findings relate to patients with epilepsy, those taking warfarin or other oral anticoagulants, and anyone developing a skin rash.

The review is excellent and clearly presented, but the discussion is possibly controversial. For example, the authors suggest that melatonin should be avoided in children because it has not been shown to alleviate jet lag in children and little is known about its safety in this group in any condition; however, 2 studies indicate a benefit in sleep-phase delay - a different circadian disorder that is common among adolescents. ${ }^{23}$ The one study in the review with a hypnotic (zolpidem) plus melatonin group showed more sleepiness and cognitive impairment. The effect of melatonin in relation to such older hypnotics as benzodiazepine is unclear. It is unknown whether melatonin is better than benzodiazepine or has an additive effect when combined with it, but the discouraging findings with zolpidem may inhibit initiation of trials to study these questions.

Finally, as the authors note, such alternative products as melatonin are poorly regulated and quality control may be problematic

Colin M Shapiro, $\mathrm{MD}, \mathrm{PhD}$ Toronto Western Research Institute Toronto, Ontario, Canada

1 Klompas M, Shapiro CM. SLAM jet lag. Thornhill, Ontario: Joli Joco Publications, 2000.

2 Kayumov L, Brown G, Jindal R, Buttoo K, Shapiro CM. A randomized, double-blind, placebo-controlled crossover study of the effect of exogenous melatonin on delayed sleep phase syndrome. Psychosom Med 2001;63:40-8.

3 Nagtegaal JE, Kerkhof GA, Smits MG, Swart AC, Van der Meer YG. Delayed sleep phase syndrome: a placebocontrolled cross-over study on the effects of melatonin administered five hours before the individual dim light melatonin onset.J Sleep Res 1998;7:135-43. 